Z Gerontol Geriat 2017 · 50:347-361

DOI 10.1007/s00391-017-1233-3

Eingegangen: 13. Januar 2017

Überarbeitet: 27. Februar 2017

Angenommen: 30. März 2017

Online publiziert: 28. April 2017

( $)$ Der/die Autor(en) 2017. Dieser Artikel ist eine Open-Access-Publikation.

\title{
Redaktion
}

M. Gosch, Zirl

H.-J. Heppner, Schwelm

W. Hofmann, Neumünster

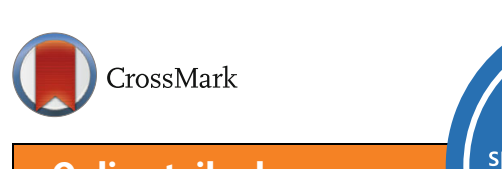

Online teilnehmen

\section{Punkte sammeln auf}

\section{CME.SpringerMedizin.de}

\section{Teilnahmemöglichkeiten}

Die Teilnahme an diesem zertifizierten Kurs ist für 12 Monate auf CME.SpringerMedizin.de möglich. Den genauen Teilnahmeschluss erfahren Sie dort.

Teilnehmen können Sie:

- als Abonnent dieser Fachzeitschrift - als e.Med-Abonnent.

\section{Zertifizierung}

Diese Fortbildungseinheit ist zertifiziert von der Ärztekammer Nordrhein gemäß Kategorie $\mathrm{D}$ und damit auch für andere Ärztekammern anerkennungsfähig. Es werden 3 Punkte vergeben.

\section{Anerkennung in Österreich}

Gemäß Diplom-Fortbildungs-

Programm (DFP) werden die auf

CME.SpringerMedizin.de erworbenen

Fortbildungspunkte von der

Österreichischen Ärztekammer 1:1 als fachspezifische Fortbildung angerechnet (§26(3) DFP Richtlinie).

\section{Kontakt}

Springer Medizin Kundenservice Tel. 08007780777

E-Mail:kundenservice@springermedizin.de

\section{CME Zertifizierte Fortbildung}

Wolfgang Löscher ${ }^{1} \cdot$ Bernhard Iglseder ${ }^{2}$ iD

${ }^{1}$ Arbeitsgruppe Neurophysiologie, Spezialbereich neuromuskuläre Erkrankungen, Universitätsklinik für Neurologie, Medizinische Universität Innsbruck, Innsbruck, Österreich

${ }^{2}$ Universitätsklinik für Geriatrie, Christian-Doppler-Klinik, Universitätsklinikum Salzburg, Paracelsus Medizinische Privatuniversität, Salzburg, Österreich

\section{Polyneuropathie im Alter}

\section{Zusammenfassung}

Das periphere Nervensystem ist im Laufe des Alternsprozesses Veränderungen, wie Abschwächung von Muskeleigenreflexen und Propriozeption, unterworfen. Davon abzugrenzen sind Polyneuropathien (PNP) als krankhafte Erscheinungen, deren Ursachen mit denen in jüngeren Lebensabschnitten übereinstimmen. Neben metabolischen, immunmediierten, hereditären, toxischen und infektiösen Ätiologien können PNP Ausdruck von Systemerkrankungen sein. Beim älteren Patienten scheinen sie im Zusammenhang mit Diabetes, monoklonalen Gammopathien und Malignomen häufiger aufzutreten. Mithilfe eines systematischen Zugangs wird in der Mehrzahl der Fälle die spezifische Diagnose gestellt. Bei alten Menschen tragen PNP zur Beeinträchtigung der Mobilität bei; daher sind ihre funktionellen Fähigkeiten abzuklären. Therapeutische Maßnahmen adressieren primär die Grundkrankheit und den neuropathischen Schmerz. Physiotherapie und Maßnahmen zum Erhalt der Alltagsfähigkeit ergänzen das Spektrum.

\section{Schlüsselwörter}

Peripheres Nervensystem · Neuropathischer Schmerz · Diabetes · Guillain-Barré-Syndrom $\cdot$ Paraneoplastische Polyneuropathie 


\section{Lernziele}

Polyneuropathien (PNP) sind häufige Erkrankungen alter Menschen und oft schwierig abzuklären. Nach Lektüre dieses Beitrags ...

- haben Sie einen Überblick über physiologische Altersveränderungen des peripheren Nervensystems (PNS) und Kenntnisse über die Ätiologie von PNP gewonnen.

- verfügen Sie über einen strukturierten Zugang zum Krankheitsbild PNP anhand von Anamnese, klinisch-neurologischer Untersuchung und Hilfsbefunden.

- wissen Sie um die Bedeutung medikamentöser Therapiemaßnahmen im Kontext der Polypharmakotherapie.

- können Sie die Auswirkungen der Erkrankung auf die Alltagsfähigkeit der Betroffenen beurteilen, um entsprechende Interventionen zu planen.

Die Zahl der sympathischen Neurone im Rückenmark nimmt pro Altersdekade um etwa $8 \%$ ab

Die Propriozeption leidet durch eine Reduktion der Mechanorezeptoren

Änderungen subkortikaler Strukturen beeinflussen die Motorik

\section{Altersassoziierte Veränderungen des peripheren Nervensystems}

Das PNS ist im Laufe des Alternsprozesses Veränderungen unterworfen. Bereits 1913 postulierte Oppenheim eine vorwiegend durch sensible Reizerscheinungen gekennzeichnete „Polyneuritis senilis“. Im Jahr 1970 wurde an Einzelfaserpräparaten von Hautnervenbiopsaten >60-jähriger Personen eine zunehmende Zahl abnormer Fasern mit Zeichen der Waller-Degeneration, segmentaler Demyelinisierung sowie Abnormitäten und Variation der internodalen Distanz nachgewiesen [1]. Auch die Zahl der sympathischen Neurone im Rückenmark nimmt pro Altersdekade um etwa $8 \% \mathrm{ab}$ [2]. Eine Abschwächung der Muskeleigenreflexe, deutlicher an den unteren Extremitäten mit Akzentuierung nach distal, imponiert als klinisches Korrelat: Der Achillessehnenreflex ist bei $38 \%$ der >65-Jährigen nicht auslösbar; die Reflexe der oberen Extremitäten fehlen lediglich bei $5 \%[3]$.

Auch das Berührungsempfinden zeigt eine zunehmend höhere Schwelle; die Propriozeption leidet durch eine Reduktion der Mechanorezeptoren in Gelenken und Haut $[4,5]$. Ein reduzierter Vibrationssinn, besonders distal an den Beinen, wurde bei $71 \%$ einer gesunden Altersgruppe ohne funktionelle Einschränkungen nachgewiesen [3].

Diese Veränderungen führen in teils kausalem Zusammenspiel mit der Abnahme der Muskelmasse (Sarkopenie) zum Verlust an Muskelkraft. Änderungen im frontalen und im präfrontalen Kortex haben Auswirkungen auf Handlungsplanung und Motivation; Änderungen subkortikaler Strukturen beeinflussen die Motorik: Es resultieren funktionelle Einschränkungen, reduzierte Mobilität und erhöhtes Sturzrisiko [5, 6].

\section{Polyneuropathy in the elderly}

\begin{abstract}
The peripheral nervous system is subject to changes during the ageing process, e.g. deep tendon reflexes decrease, as does proprioception. Polyneuropathies, on the other hand, need to be distinguished from age-related changes as independent diseases with etiologies similar to those at younger ages. Etiologies includes metabolic disorders, primary inflammatory polyneuropathies, and systemic disorders. Neuropathies associated with diabetes, malignancy, and monoclonal gammopathies appear to be more common in older patients. Using a systematic approach, it is possible to establish a specific diagnosis in the majority of cases. Since polyneuropathies contribute to reduced mobility in the elderly, an assessment of functional skills is mandatory. Polyneuropathy therapy is primarily based on the treatment of underlying conditions and neuropathic pain management. Physiotherapy and rehabilitation target pain relief and maintaining activities of daily living.
\end{abstract}

\section{Keywords}

Peripheral nervous system · Neuropathic pain · Diabetes · Guillain-Barre syndrome $\cdot$ Paraneoplastic polyneuropathy 
Das Konzept des Zusammenspiels neuronaler und muskulärer Faktoren als Ursache der Muskelschwäche im Alter fand unter dem Terminus Dynapenie Eingang in die Literatur [7]. Auch Innenohr, Vestibulariskerne, Visus und kardiopulmonales System sind Altersveränderungen unterworfen, die zur Verschlechterung der Gleichgewichtsfähigkeit beitragen. Von diesen physiologischen Veränderungen müssen PNP als definierte Erkrankungen abgegrenzt werden.

\section{Definition, Epidemiologie, Ursachen}

Polyneuropathien sind generalisierte Erkrankungen des PNS. Dies umfasst die außerhalb des Zentralnervensystems (ZNS) liegenden motorischen, sensiblen und autonomen Nerven mit SchwannZellen, ganglionären Satellitenzellen, bindegewebigen Hüllstrukturen sowie die zugehörigen Blutund Lymphgefäße. Es gibt über 500 Ursachen für eine PNP. Die weltweit führende Ätiologie der PNP ist die Lepra.

Die geschätzte jährliche Inzidenz beträgt in westlichen Industrienationen 118/100.000, die Prävalenz insgesamt $1 \%$ und für $>55$-Jährige $3 \%$. Für ältere Populationen (über 65 Jahre) werden $7 \%$ angegeben. Frauen sind häufiger betroffen [8].

Die Ursachen sind mannigfaltig: Neben metabolischen, immunmediierten, hereditären, toxischen und infektiösen Ätiologien treten PNP im Rahmen von Systemerkrankungen auf. Krankheiten, die eine intensivmedizinische Therapie erfordern, können durch eine „critical illness neuropathy“ kompliziert werden.

Im Alter nimmt die Inzidenz der diabetischen Polyneuropathie (DPNP; etwa $30 \%$ der Fälle) zu. Auch die Zahl der kryptogenen axonalen Polyneuropathien (CAP) steigt deutlich an. Sie verursacht bei den >80-Jährigen $35 \%$ der Erkrankungen [9].

Mithilfe eines systematischen Zugangs kann in neuromuskulären Zentren in bis zu $75 \%$ der Fälle eine spezifische Diagnose gestellt werden. Ziel ist, über Anamnese, klinische und elektrophysiologische Untersuchung, eine Verdachtsdiagnose zu stellen und diese mithilfe spezifischer Laboruntersuchungen zu verifizieren, um die gezielte Therapie einzuleiten [10].

\section{Diagnostik}

\section{Anamnese}

\section{Allgemeine Anamnese}

Klinische Kennzeichen von PNP umfassen motorische, sensible und autonome Störungen. Muskelkrämpfe, Faszikulationen oder Myokymien zählen zu den positiven motorischen Symptomen und deuten auf immunmediierte Neuropathien hin. Schwäche, Lähmungen und Muskelatrophie zählen zu den negativen motorischen Symptomen, die Ausdruck fortgeschrittener Stadien sind. Positive sensible Symptome wie Dysästhesien und Parästhesien helfen, erworbene von hereditären PNP zu unterscheiden, da bei Letzteren positive sensible Symptome selten sind. Negative sensible Symptome treten in Form von Hypästhesien auf.

Informationen über Beginn, Dauer und zeitlichen Verlauf erlauben die weitere diagnostische Eingrenzung. Akute motorische Verlaufsformen sprechen für das Vorliegen eines GuillainBarré-Syndroms (GBS) oder einer Vaskulitis. Schubhafte Verläufe weisen auf eine chronisch inflammatorische demyelinisierende Polyneuropathie (CIDP) hin. Porphyrien oder hereditäre Neuropathien spielen im Alter eine untergeordnete Rolle, da diese Diagnosen früher gestellt werden. Viele PNP treten im Rahmen von Systemerkrankungen auf. Die gezielte Anamnese adressiert:

- Diabetes mellitus,

- chronisches Nierenversagen,

- Schilddrüsenerkrankungen,

- Lebererkrankungen,

- Malabsorption,

- Tumorerkrankungen,

- rheumatische Erkrankungen und

- „Human-immunodeficiency-virus“(HIV)-Infektion.
Für ältere Populationen (über 65 Jahre) wird eine 7 \%ige PNP-Prävalenz angegeben

Die Zahl der kryptogenen axonalen Polyneuropathien steigt im Alter deutlich an

Negative motorische Symptome sind Ausdruck fortgeschrittener Stadien

Akute motorische Verlaufsformen können das Vorliegen eines Guillain-Barré-Syndroms anzeigen 


\begin{tabular}{|c|c|c|c|c|}
\hline Kardiovaskulär & ZNS wirksam & $\begin{array}{l}\text { Chemotherapeutika, } \\
\text { Immunsupressiva }\end{array}$ & $\begin{array}{l}\text { Virostatika, Antibio- } \\
\text { tika }\end{array}$ & Andere \\
\hline Amiodaron & Phenytoin & Vincristin & Nukleosidanaloga & Colchicin \\
\hline Enalapril $^{\mathrm{a}}$ & Thalidomid & Cisplatin & Isoniazid & Penicillamin \\
\hline Hydralazin $^{\mathrm{a}}$ & Lithium & Oxaliplatin & Ethambutol & Disulfiram \\
\hline Perhexilin ${ }^{a}$ & Ganglioside $^{a}$ & Taxoide & Metronidazol & Gold $^{\mathrm{a}}$ \\
\hline \multirow[t]{3}{*}{ Statine $^{\mathrm{a}}$} & \multirow[t]{3}{*}{ Amitriptylin ${ }^{\mathrm{a}}$} & Suramin & Nitrofurantoin ${ }^{a}$ & Cimetidin $^{\mathrm{a}}$ \\
\hline & & Tacrolimus $^{\mathrm{a}}$ & Chloramphenicol $^{\mathrm{a}}$ & Pyridoxin $^{a}$ \\
\hline & & $\begin{array}{l}\text { Interferon alpha } \\
\text { Bortezomib }\end{array}$ & $\begin{array}{l}\text { Chloroquin }^{\mathrm{a}} \\
\text { Chinolone }^{\mathrm{a}}\end{array}$ & $\begin{array}{l}\text { Amphetamine }^{a} \\
\text { Allopurinol }^{a}\end{array}$ \\
\hline
\end{tabular}

Ebenso sind Fragen nach Alkohol- und Drogenkonsum, Diätgewohnheiten und möglicher Exposition gegenüber toxischen Substanzen (Lösungsmittel, Pestizide, Schwermetalle) abzuklären.

\section{Medikamentenanamnese}

Zahlreiche Medikamente können eine PNP verursachen [11]; daher ist die exakte Medikamentenanamnese obligat. Neben bekannten, potenziell PNP-induzierenden Medikamenten - z. B. antineoplastischen Chemotherapeutika, Isoniazid oder Thalidomid - wurden rezent weitere Substanzgruppen beschrieben: Statine können nach langem Gebrauch sensible und sensomotorische PNP auslösen [12], die nach Absetzen des Medikaments reversibel sind. Bortezomib verursacht nur teilweise reversible schmerzhafte sensomotorische PNP [13]. Linezolid kann nach längerer Anwendung eine schmerzhafte sensomotorische PNP und eine Optikusneuropathie auslösen, v. a. in Kombination mit selektiven Serotonin-Wiederaufnahmehemmern („selective serotonin reuptake inhibitors", SSRI, [14]). Auch Rituximab oder Tumor-Nekrose-Faktor-Blocker können in Einzelfällen Neuropathien bewirken (•Tab. 1).

\section{Klinische Untersuchung}

Die klinische Untersuchung erfasst:

- Reflexstatus,

- Spitz-/Stumpfdiskrimination,

- Kalt- und Warmempfinden sowie

- Vibrationsempfinden.

Das anatomische Verteilungsmuster und das Ausmaß der Beteiligung des motorischen, sensiblen und autonomen Nervensystems werden bestimmt. Mononeuropathien, d. h. isolierte Ausfälle im Verlauf nur eines peripheren Nerven, sprechen für einen lokalen Prozess, wie z. B. Trauma, Kompression, neoplastische Infiltration oder eine vaskuläre Läsion. Die Mononeuritis multiplex (multiple Mononeuropathie) findet sich bei Vaskulitiden oder diabetischer Mikroangiopathie. Asymmetrische rein motorische Defizite weisen auf eine multifokale Motoneuropathie mit Leitungsblock (MMN) oder eine Motoneuronerkrankung hin. Während die meisten Neuropathien symmetrisch vorwiegend die unteren Extremitäten betreffen, sind bei MMN, bei Porphyrie, familiärer Amyloidneuropathie Typ 2 und der seltenen Bleineuropathie die motorischen Nerven der oberen Extremitäten stärker oder isoliert betroffen. Die typische längenabhängigen Neuropathien, z. B. äthylische Neuropathie und klassische DPNP, beeinträchtigen die unteren Extremitäten distal stärker. Im Gegensatz dazu finden sich bei CIDP, osteosklerotischem Myelom und diabetischer Amyotrophie proximale und distale Paresen.

Die Prüfung aller sensiblen Qualitäten erlaubt die Beurteilung der Funktion myelinisierter und unmyelinisierter Fasern: Taktile Reize, Lagesinn, Vibration und Muskeleigenreflexe werden über myelinisierte, Schmerz und Temperatur über unmyelinisierte Fasern geleitet. Bei den meisten PNP sind beide Fasertypen betroffen. Isoliert die unmyelinisierten Fasern betreffende Neuropathien finden sich bei der Amyloidneuropathie, der hereditären sensiblen und autonomen 
Tab. 2 Elektrophysiologische Klassifizierung von Neuropathien

Uniform demyelinisierend

Segmental demyelinisierend (motorisch mit/ohne Leitungsblock - sensibel)

Motorisch axonal (symmetrisch - asymmetrisch)

Sensibel axonal (symmetrisch - asymmetrisch)

Sensomotorisch axonal (symmetrisch asymmetrisch)

Autonom

\section{Elektrophysiologie}

Im Alter findet sich eine zunehmende Abnahme der sensiblen Nervenleitgeschwindigkeiten und -amplituden [15]. Der elektrophysiologischen Untersuchung kommen diagnostisch verschiedene Funktionen zu: Sind aus Anamnese und klinischer Untersuchung eine PNP und deren Ätiologie gesichert, kann die Elektrophysiologie mithilfe von Neurographie (Nervenleitgeschwindigkeit, NLG), Elektromyographie (EMG) und autonomen Tests Ausmaß und Verteilung der Nervenschädigung sowie die Beteiligung der verschiedenen Nervenfasertypen objektivieren.

Von größerer Bedeutung ist die Elektrophysiologie, wenn Art und Ätiologie der Neuropathie unklar sind, da Informationen über die zugrunde liegende Nervenschädigung gewonnen werden können. Demyelinisierende Neuropathien werden von axonalen unterschieden. Subklinische Beteiligungen von motorischen, sensiblen oder autonomen Fasern können objektiviert werden. Demyelinisierungen können uniform sein, wie bei der häufigsten hereditären sensomotorischen PNP Charcot-Marie Tooth Typ 1, segmental bei CIDP, an typischen Engstellen bei der hereditären Neuropathie mit Druckparesen. Leitungsblöcke bei normaler sensibler Neurographie finden sich bei der MMN. Symmetrische, distal betonte axonale Neuropathien lassen sich von asymmetrischen multiplen Mononeuropathien abgrenzen, ebenso wie Motoneuronerkrankungen von motorischen Neuropathien.

Bei Sensibilitätsstörungen weisen erhaltene und unauffällige sensible Potentiale auf eine präganglionäre Schädigung. Das Ergebnis der elektrophysiologischen Untersuchung erlaubt die Zuordnung zu einer von 5 möglichen pathophysiologischen Klassen (• Tab. 2), die, abhängig vom zeitlichen Verlauf, die weitere Abklärung erleichtern [16].

\section{Laboruntersuchungen}

Die labormedizinische Abklärung richtet sich nach Anamnese und klinischen Symptomen, Häufigkeit spezifischer Ursachen, assoziierten Symptomen und elektrophysiologischer Klassifizierung [17].

In Mitteleuropa sind bei klinisch typischer symmetrischer distal betonter sensomotorischer PNP die Bestimmungen von Nüchternblutzucker und $\mathrm{HbA}_{1 \mathrm{c}}$ die Untersuchungen der 1. Wahl, da Diabetes mellitus eine der häufigsten Ursachen für PNP darstellt. Weiterhin werden die Leberenzyme untersucht, da auch übermäßiger Alkoholkonsum zu den häufigsten Ursachen für PNP zählt. Ebenso gilt dies für das chronische Nierenversagen, was die Testung der Nierenparameter erforderlich macht. Sind diese Befunde negativ, ist die weitere Abklärung entsprechend Verlauf und elektrophysiologischer Klassifizierung der PNP durchzuführen (• Tab. 3 und 4).

\section{Nervenbiopsie}

Verdacht auf Vaskulitis, Amyloidose oder neoplastische Infiltration sind die wichtigsten Indikationen einer Biopsie [18]. Bei Verdacht auf Vaskulitis erhöht eine gleichzeitig durchgeführte Muskelbiopsie die diagnostische Sensitivität. In der Regel wird der N. suralis am Unterschenkel biopsiert, alternativ der N. peronaeus superficialis. Ohne begründete Verdachtsdiagnose leisten Biopsien nur in ca. $20 \%$ einen diagnostischen Beitrag.
Eine Schädigung der myelinisierten Fasern führt zu ataktischen Neuropathien

Demyelinisierende Neuropathien werden mithilfe der Elektrophysiologie von axonalen unterschieden

Bei Sensibilitätsstörungen weisen unauffällige sensible Potenziale auf eine präganglionäre Schädigung hin

In Mitteleuropa stehen Diabetesparameter bei symmetrischer distal betonter sensomotorischer PNP an 1. Stelle der Laboruntersuchung

Bei Verdacht auf Vaskulitis erhöht eine gleichzeitig durchgeführte Muskelbiopsie die diagnostische Sensitivität 


\begin{tabular}{|c|c|c|c|}
\hline Axonal sensomotorisch & Axonal sensibel & Axonal motorisch & Demyelinisierend \\
\hline Lumbalpunktion $^{a}$ & ANA, ANCA ${ }^{a}$ & Lumbalpunktion $^{\mathrm{a}}$ & Lumbalpunktion $^{\mathrm{a}}$ \\
\hline Porphyrine (Urin, Stuhl) ${ }^{\mathrm{b}}$ & SS-A AK, SS-B AK ${ }^{\mathrm{a}}$ & $\begin{array}{l}\text { 24-h-Urin (Arsen, } \\
\text { Thallium) }\end{array}$ & Borrelien (Liquor) ${ }^{a}$ \\
\hline Borrelien (Liquor) ${ }^{b}$ & Antineuronale $\mathrm{AK}^{\mathrm{a}}$ & Porphyrine (Urin, Stuhl) ${ }^{b}$ & HIV $^{\mathrm{a}}$ \\
\hline ANA, ANCA $^{\text {b }}$ & Tumorscreening $^{\mathrm{a}}$ & $\begin{array}{l}\text { PNS-AK }{ }^{\text {b }} \text { (z. B. Ganglio- } \\
\text { sid-AK) }\end{array}$ & 24-h-Urin (Arsen, Thallium) \\
\hline $\begin{array}{l}\text { PNS-AK }{ }^{\text {b }} \text { (z. B. Ganglio- } \\
\text { sid-AK) }\end{array}$ & HIV $^{\mathrm{a}}$ & Tumorscreening $^{c}$ & Hepatitis $B+C^{b}$ \\
\hline \multirow[t]{4}{*}{ Campylobacter jejuni $^{\text {b }}$} & $\begin{array}{l}\text { AK gegen das Gan- } \\
\text { gliosid GQ1 } \mathrm{b}^{\mathrm{b}}\end{array}$ & \multirow[t]{4}{*}{ Campylobacter jejuni $^{\text {b }}$} & ANCA, ANA ${ }^{b}$ \\
\hline & \multirow{3}{*}{$\begin{array}{l}\text { Speicheldrüsen- } \\
\text { biopsie }^{\text {b }}\end{array}$} & & Anti-dsDNA ${ }^{b}$ \\
\hline & & & $\begin{array}{l}\text { PNS-AK ( } \text { (z. B. Ganglio- } \\
\text { sid-AK) }\end{array}$ \\
\hline & & & Campylobacter jejuni ${ }^{b}$ \\
\hline \multicolumn{4}{|c|}{ 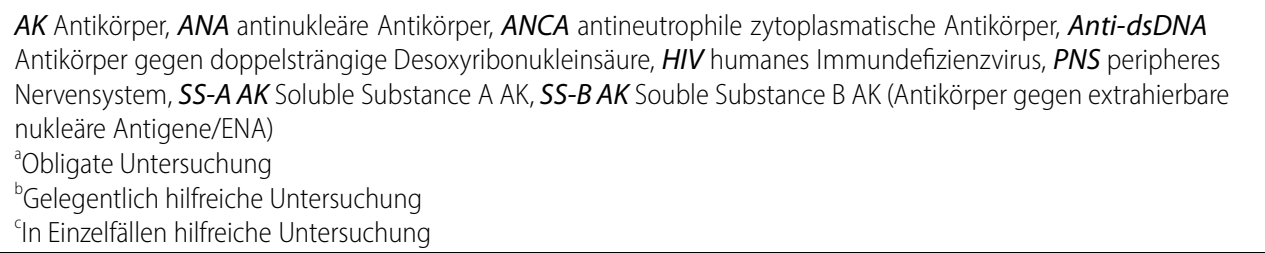 } \\
\hline
\end{tabular}

Die Prävalenz einer DPNP wird bei 70- bis 79-Jährigen Diabetes-Patienten mit $44 \%$ angegeben

Am häufigsten ist die distal symmetrische sensibel betonte Neuropathie

Die diabetische Ophthalmoplegie geht mit Ausfällen der Hirnnerven III, IV und VI einher

\section{Häufige Polyneuropathien des höheren Lebensalters}

\section{Diabetische Polyneuropathien (DPNP)}

Diabetische Polyneuropathien treten bei $>50 \%$ der Diabetespatienten $>60$ Jahre auf. Sowohl die sensomotorische diabetische Neuropathie als auch die autonome diabetische Neuropathie nehmen im Alter zu: Bei Diabetes-Patienten wird die Prävalenz einer DPNP bei 20- bis 29Jährigen mit $5 \%$, bei 70 - bis 79-Jährigen dagegen mit $44 \%$ angegeben. Die Prävalenz der symptomatischen diabetischen Neuropathie beträgt bei Typ-1- und Typ-2-Diabetes 15-30\%, die der asymptomatischen bis $66 \%$ [19].

Am häufigsten ist die distal symmetrische, sensibel betonte und zusätzlich autonome Neuropathie. Dabei kommt es distal betont zu strumpfförmigen Parästhesien. Häufig finden sich in der Folge Pallhypästhesie (reduzierte Wahrnehmung von Vibration, Berührung und Druck) und Hypalgesie (reduzierte Schmerzwahrnehmung). Charakteristisch sind auch Dehnungs- und Druckschmerzen an den Nervenendpunkten in den Muskeln. Im Verlauf kann es zu motorischen Ausfällen, z. B. zum Verlust der Muskel- und Sehnenreflexe, und schließlich zu schlaffen Lähmungen kommen. Autonome Symptome umfassen orthostatische Dysregulation, Obstipation oder Diarrhö und Anhidrose.

Die multifokalen Neuropathien (diabetische Ophthalmoplegie, Radikulopathie und Amyotrophie) sind vorwiegend Manifestationen des fortgeschrittenen Alters. Die diabetische Ophthalmoplegie mit Ausfällen der Hirnnerven III, IV und VI setzt oft mit heftigen orbitalen Schmerzen ein, gefolgt von Doppelbildern. Am häufigsten sind der N. abducens (VI. Hirnnerv) und N. oculomotorius (III. Hirnnerv) betroffen. Die Pupillenreaktion bleibt meist erhalten. Häufig remittieren die Symptome innerhalb von 4 bis 6 Wochen. Ischämische Prozesse der Vasa nervorum und des Mesencephalons werden ursächlich angeschuldigt.

Die diabetische Radikulopathie ist durch plötzlich auftretende Schmerzen charakterisiert, die gürtelförmig ein- oder beidseitig von der Wirbelsäule zur Brust- oder zur Bauchregion ziehen. In den betroffenen Segmenten finden sich Hypästhesie und Hypalgesie, manchmal ein Ausfall der Schweißsekretion. Sind motorische Fasern betroffen, imponiert eine Lähmung der Bauchwandmuskulatur. Die Prognose ist ungewiss; häufig persistieren Schmerzen über lange Zeiträume. 
Tab. 4 Laboruntersuchungen bei subakut-chronischen Polyneuropathien ( $>4$ bis 8 Wochen Krankheitsverlauf), unterteilt nach klinischem Verlauf und elektrophysiologischer Klassifizierung

\begin{tabular}{|c|c|c|c|}
\hline Axonal sensomotorisch & Axonal sensibel & Axonal motorisch & Demyelinisierend \\
\hline Glucose, $\mathrm{HbA}_{1 c}{ }^{\mathrm{a}}$ & Glucose, $\mathrm{HbA}_{1 c}{ }^{\mathrm{a}}$ & SIEP/IFX & Lumbalpunktion $^{\mathrm{a}}$ \\
\hline Leberfunktionsproben $^{\mathrm{a}}$ & ANA, ANCA ${ }^{a}$ & SMN-Mutationen $^{\mathrm{b}}$ & HIV $^{a}$ \\
\hline Nierenfunktionsproben ${ }^{a}$ & SS-A AK, SS-B AK & "CAG repeats ${ }^{\text {"b }}$ & Glucose, $\mathrm{HbA}_{1 c}{ }^{\mathrm{a}}$ \\
\hline $\mathrm{BB}+$ Diff $^{\mathrm{a}}$ & Vitamin $\mathrm{B}_{12}(\mathrm{Hcy}, \mathrm{Mmal})^{\mathrm{a}}$ & Lumbalpunktion $^{c}$ & SIEP/IFIX \\
\hline SIEP/IFIX & SIEP/IFIX & \multirow{11}{*}{$\begin{array}{l}\text { PNS-AK' (z. B. } \\
\text { Gangliosid-AK) }\end{array}$} & Bence-Jones-Protein $^{\mathrm{a}}$ \\
\hline Vitamin $\mathrm{B}_{12}(\mathrm{Hcy}, \mathrm{Mmal})^{\mathrm{a}}$ & $\mathrm{SD}+\mathrm{TSH}^{\mathrm{a}}$ & & Skelettröntgen ${ }^{\mathrm{b}}$ \\
\hline $\mathrm{SD}, \mathrm{TSH}^{\mathrm{a}}$ & $\mathrm{HIV}^{\mathrm{b}}$ & & $\begin{array}{l}\text { PNS-AK (z. B. Ganglio- } \\
\text { sid-AK) }\end{array}$ \\
\hline$H_{I V}$ & $\begin{array}{l}\text { PNS-AK }{ }^{b} \text { (z. B. Ganglio- } \\
\text { sid-AK) }\end{array}$ & & PMP22, MPZ, Cx $22^{\mathrm{b}}$ \\
\hline ANA, ANCA ${ }^{b}$ & Vitamin $E^{c}$ & & Borrelien (Liquor) $2^{\mathrm{a}}$ \\
\hline SS-A, SS-B ${ }^{b}$ & Speicheldrüsenbiopsie ${ }^{c}$ & & VLCFA $^{c}$ \\
\hline Triglyzeride, Cholesterin ${ }^{c}$ & Antineuronale $\mathrm{AK}^{\mathrm{c}}$ & & Phytansäure ${ }^{c}$ \\
\hline $\mathrm{HDL}, \mathrm{LDL}^{\mathrm{c}}$ & \multirow[t]{4}{*}{ Tumorscreening ${ }^{c}$} & & \multirow[t]{4}{*}{ Arylsulfatase $A^{c}$} \\
\hline $\begin{array}{l}\text { PNS-AK ' (z. B. Ganglio- } \\
\text { sid-AK) }\end{array}$ & & & \\
\hline VLCFA $^{c}$ & & & \\
\hline Genetische Testungen*c & & & \\
\hline \multicolumn{4}{|c|}{ 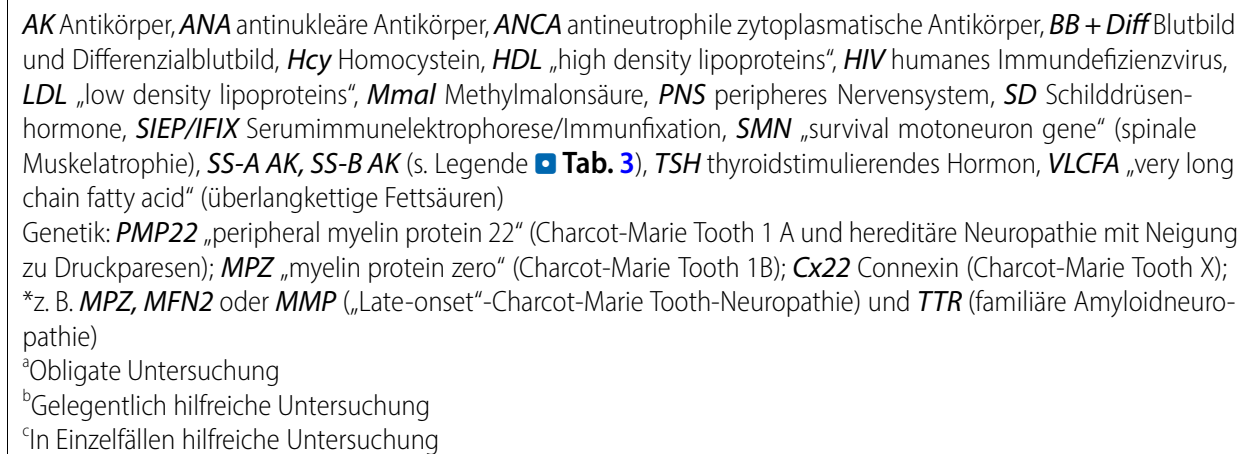 } \\
\hline
\end{tabular}

Die diabetische Amyotrophie (oder diabetische lumbosakrale Radikuloplexoneuropathie) entwickelt sich überwiegend bei Typ-2-Diabetes und tritt meist jenseits des 50. Lebensjahres auf [20]. Männer erkranken häufiger. Es kommt zu akut einsetzenden Schmerzen, die von lumbal zur Ventralseite eines Oberschenkels ziehen. Rasch entwickeln sich Lähmungen und Atrophien der Beckengürtel- und Oberschenkelmuskel, begleitet von einem Ausfall des Patellarsehnenreflexes. Sensibilitätsstörungen stehen im Hintergrund. Die Differenzialdiagnose zu radikulären Ausfällen kann schwierig sein und bildgebende Verfahren notwendig machen. Die Prognose ist variabel; Schmerzen können wochenlang anhalten. Da die histologische Untersuchung von Nervenbiopsaten eine epineurale Vaskulitis zeigte [20], sind Behandlungsversuche mit Steroiden oder Immunglobulinen vertretbar.

\section{Polyneuropathien bei monoklonalen Gammopathien}

Im Alter finden sich vermehrt Paraproteinämien (monoklonale Gammopathien): Bei $3 \%$ der $>70$-Jährigen sowie bei $10 \%$ der PNP-Patienten dieser Altersstufe ist eine monoklonale Gammopathie nachweisbar [21]. Lediglich $30 \%$ dieser Patienten entwickeln eine systemische Erkrankung wie ein multiples Myelom oder einen M. Waldenström; bei allen anderen spricht man von einer monoklonalen Gammopathie unklarer Signifikanz (MGUS). Bezüglich der Immunglobulinklassen überwiegt mit $>50 \%$ der IgM-Typ. Bei $>50 \%$ der IgM-MGUS-PNP lassen sich Antikörper gegen myelinassoziiertes Glykoprotein (MAG) nachweisen. Die MGUS-PNP verlaufen langsam progredient distal symmetrisch; initial dominieren sensible Symptome. Der Nachweis des mo-

Bei $>50 \%$ der IgM-MGUS-PNP lassen sich Antikörper gegen myelinassoziiertes Glykoprotein nachweisen 
noklonalen Immunglobulins erfolgt mithilfe der Serumelektrophorese und Immunfixation. Die Differenzierung zwischen MGUS und anderen Formen erfordert eine hämatologische Abklärung. Im Fall der PNP infolge eines multiplen Myeloms steht die Behandlung der Grundkrankheit im Vordergrund. Die MGUS-PNP werden wie eine CIDP behandelt (s. Abschn. „Kausale Therapie“). Insgesamt schlechter sprechen IgM-Gammopathien auf die Therapie an.

\section{Entzündliche Polyneuropathien}

\section{Guillain-Barré-Syndrom}

Die akute inflammatorische demyelinisierende Polyradikuloneuropathie (AIDP), nach den Erstbeschreibern als Guillain-Barré-Syndrom (GBS) bezeichnet, zeigt eine Zunahme der Inzidenz mit steigendem Alter [22]. Klinisch imponiert eine progrediente Muskelschwäche an mehr als einer Extremität; Fazialisparesen, Dysphagie und externe Ophthalmoplegie erweitern das Bild. Die akut beginnenden Paresen erreichen ihr Maximum innerhalb von 4 Wochen und sind relativ symmetrisch verteilt. Sensible Symptome sind meistens nur gering ausgeprägt, allerdings ist das Vorhandensein von Schmerzen kein Ausschlussgrund. Autonome Funktionsstörungen wie Tachykardie, Arrhythmie, posturale Hypotension, arterielle Hypertension und vasomotorische Dysregulation können den Verlauf komplizieren. Es findet sich eine erhöhte Liquor-Protein-Konzentration bei normaler oder minimal erhöhter Zellzahl. Therapeutisch wird bei alten Menschen die Gabe von Immunglobulinen wegen der schonenderen Applikation gegenüber der Plasmapherese bevorzugt. Autonome Symptome oder eine Beteiligung der Atemmuskulatur können eine Intensivbehandlung erforderlich machen.

\section{Chronisch inflammatorische demyelinisierende Polyneuropathie}

Die CIDP verläuft im Alter meist chronisch progredient $>2$ Monate

Meist handelt es sich bei den Vaskulitiden um immunmediierte Formen

Die Pathogenese der alkoholisch bedingten PNP beruht vorwiegend auf der direkten neurotoxischen Wirkung

Die chronische inflammatorische demyelinisierende Polyneuropathie (CIDP) verläuft im Alter meist chronisch progredient über $>2$ Monate, seltener schubförmig ( $\geq 2$ Schübe, [23]). Neben sensomotorischen Defiziten an mehr als einer Extremität imponieren abgeschwächte oder fehlende Muskeleigenreflexe. Im Liquor ist eine allenfalls geringe Pleozytose bei erhöhter Proteinkonzentration nachzuweisen; die elektrophysiologischen Kriterien wurden bereits erwähnt.

\section{Polyneuropathien bei Vaskulitis}

Vaskulitiden stellen eine heterogene Krankheitsgruppe dar; dabei kommt es zu einer Entzündung mit struktureller Schädigung der Gefäßwand. Die Prävalenz nimmt mit dem Alter zu [24, 25]. Direkt erregerbedingte Vaskulitiden (u. a. Borrelien, Varicella-zoster-, Zytomegalievirus) sind selten; meist handelt es sich um immunmediierte Formen. Häufigste Ursache ist die Panarteriitis nodosa. Die klinische Manifestation ist relativ uniform mit Schmerzen, Sensibilitätsstörungen und Paresen im Versorgungsgebiet unterschiedlicher peripherer Nerven im Sinne einer Mononeuritis multiplex. Am häufigsten sind N. peronaeus, N. tibialis und N. ulnaris betroffen; auch eine distal symmetrische PNP ist möglich. Der Verlauf ist meist akut oder subakut; die Therapie erfolgt mithilfe von Immunsuppressiva.

\section{Toxische Polyneuropathien}

Eine Vielzahl neurotoxischer Substanzen kann zu PNP führen [11]. Im Alter stehen Pharmaka im Vordergrund (s. Abschn. „Anamnese“), während der Alkoholmissbrauch eher zurücktritt. Die Pathogenese der alkoholisch bedingten PNP beruht vorwiegend auf einer direkten neurotoxischen Wirkung. Auch ein ernährungsbedingter Vitaminmangel spielt eine Rolle. Die alkoholische Polyneuropathie tritt meist als schmerzhafte distal symmetrische, vorwiegend sensible Störung auf. In der Folge können distal symmetrische atrophe Paresen, trophische Störungen und Ödeme hinzukommen [26]. Elektrophysiologisch finden sich axonale Schäden. Entscheidend ist die Abstinenz, wobei zusätzlich Vitamin $B_{1}$ verordnet wird, um weitere alkoholassoziierte Erkrankungen $\mathrm{zu}$ vermeiden. 


\section{Polyneuropathien durch Vitaminmangel}

Eine Mangelernährung als Ursache von Hypovitaminosen findet sich im Alter bei gestörter enteraler Resorption. Meistens besteht ein Vitamin-B-Mangel. Chronische Darm- oder Lebererkrankungen können zu einem Vitamin- $\mathrm{B}_{1}$ (Thiamin)-Mangel führen. Auch Mangelerscheinungen von Vitamin $\mathrm{B}_{6}$ (Pyridoxin) und Vitamin $\mathrm{B}_{12}$ (Cobalamin) sind bekannt [11]. Die Substitution kann zur Remission führen, die allerdings nicht immer vollständig ist.

\section{Paraneoplastische Neuropathien}

Im Verlauf maligner Tumorerkrankungen kann das PNS beteiligt sein. Eine lokale Tumorinfiltration kann Hirnnerven, einzelne oder mehrere Nervenwurzeln im Plexus brachialis oder lumbosacralis sowie periphere Nerven treffen. Antineoplastische Therapeutika können Nebenwirkungen entfalten, die periphere Nerven schädigen [11].

Gelegentlich treten PNP bei Tumorpatienten auch ohne Medikamentenexposition oder Tumorinfiltration auf, in diesen Fällen liegt eine paraneoplastische Erkrankung vor [27], die zeitlich der Entdeckung der Tumorerkrankung vorausgehen kann. Häufigste Ursache ist das kleinzellige Bronchialkarzinom, gefolgt von Mamma- und Ovarialkarzinomen sowie Lymphomen. Die häufigste paraneoplastische Form ist die sensible PNP, die meist distal symmetrisch beginnende Dysästhesien, Hypästhesien und Schmerzen bedingt. Nachfolgend kann eine sensible Ataxie auftreten. Pathologische Veränderungen werden hauptsächlich an den Spinalganglienneuronen gefunden, was zur Bezeichnung Neuronopathie geführt hat.

Bei mehr als $90 \%$ der Patienten können im Serum antineuronale Antikörper nachgewiesen werden. Selten kann eine autonome PNP als Paraneoplasie mit orthostatischer Hypotension, Urinretention, Obstipation und Pupillenstörungen auftreten. Die onkologische Behandlung stellt die zentrale Maßnahme dar; Immunmodulation und symptomatische Therapieformen ergänzen das Spektrum [28].

\section{Morbus Parkinson}

Rezente Beobachtungen legen eine Assoziation von M. Parkinson und PNP nahe. Die Höhe der L-Dopa-Dosierungkorreliert mit dem Schweregrad der PNP [29]. Erhöhte Homocysteinund Methylmalonsäurespiegel sind Zeichen eines Vitamin--B12-Mangels. Die Substitution führt zumindest zu einer Stabilisierung der Symptomatik.

\section{Herpes zoster}

Der Herpes zoster ist eine Erkrankung des hohen Alters. Die Manifestation bevorzugt das Areal des N. trigeminus und die thorakalen Dermatome. Im Alter besteht ein erhöhtes Risiko zur postherpetischen Neuralgie, deren Behandlung sich an symptomatischen Kriterien orientiert.

\section{Therapie}

Für wenige Formen der PNP stehen kausale Ansätze zu Verfügung; die symptomatische Therapie orientiert sich am Beschwerdebild. Therapie der Wahl ist die Behandlung der Grunderkrankung. Allerdings bessern sich die Beschwerden häufig langsam und nur unvollständig.

\section{Kausale Therapie}

Kausale Therapiemöglichkeiten bestehen für immunmediierte und infektiöse PNP sowie bei Systemerkrankungen. Akute immunmediierte PNP wie das GBS sprechen auf intravenöse Immunglobuline (IVIG) oder Plasmapherese an. Chronische immunmediierte PNP wie CIDP lassen sich mit Prednisolon, IVIG und Plasmapherese behandeln. Die Prednisolonstandarddosis beträgt $1 \mathrm{mg} / \mathrm{kgKG}$ für 2 bis 4 Wochen; anschließend wird auf die Hälfte der Dosis reduziert. Diese wird beibehalten, bis sich die Muskelkraft des Patienten normalisiert oder die Besserung ein Plateau erreicht hat. Danach erfolgt je nach klinischen Symptomen langsam die weitere Dosisreduktion.
Die häufigste paraneoplastische Form ist die sensible PNP

Bei mehr als $90 \%$ der Patienten können im Serum antineuronale Antikörper nachgewiesen werden

Die Höhe der L-Dopa-Dosierung bei M. Parkinson korreliert mit dem Schweregrad der PNP

Therapie der Wahl ist die Behandlung der Grunderkrankung 
Die MMN kann sich unter einer Steroidtherapie verschlechtern

Die Hemmung von CYP2D6 ist unter dem Aspekt einer Polypharmakotherapie zu beachten

Unter SSRI- und SNRI-Gabe ist auf eine Verlängerung der QT-Zeit zu achten

Carbamazepin kann eine Hyponatriämie auslösen
Eine Ausnahme stellt die MMN dar, die sich unter Steroidtherapie verschlechtern kann. Azathioprin, Mycophenolat-Mofetil, Cyclophosphamid, Chlorambucil, Cyclosporin A, Tacrolimus und Rituximab wurden in Einzelfällen erfolgreich angewandt. Die Behandlung von vaskulitischen Neuropathien basiert auf Steroiden und Immunsuppressiva [30].

Bei einer Steroidtherapie, die für länger als 3 Monate geplant ist, sollte eine Osteoporoseprophylaxe angeboten werden. In der Behandlung von infektiösen Neuropathien/Polyradikuloneuropathien ist die Indikation zur Antibiotikatherapie der Neuroborreliose erwähnenswert [31].

\section{Symptomatische Therapie}

Schmerzhafte Sensibilitätsstörungen sind häufig die im Vordergrund stehenden Symptome. Der neuropathische Schmerz wird durch folgende Mechanismen ausgelöst:

- ektope Aktivität in sensitivierten C-Fasern,

- Rekrutierung ruhender Schmerzrezeptoren,

- spontane Aktivität in Spinalganglien und

- sekundäre zentrale Adaptationen.

Die resultierende Sensitivierung zentraler sensibler Neurone beruht auf vermehrter Natriumkanalexpression, vermehrterglutamaterger Stimulation von N-Methyl-D-Aspartat(NMDA)-Rezeptoren sowie serotonergen und noradrenergen Mechanismen [32].

\section{Pharmakotherapie}

Zahlreiche Medikamente wurden in der Behandlung neuropathischer Schmerzen in randomisierten placebokontrollierten Studien untersucht [33]. Entsprechend der Pathophysiologie werden Antidepressiva, natriumkanal- und kalziumkanalblockierende Antiepileptika sowie Opiate mit zusätzlichen monoaminergen Wirkmechanismen verwendet. Mehrheitlich wurde die Wirksamkeit dieser Substanzen bei schmerzhafter DPNP und bei postherpetischer Neuralgie untersucht. Einzelne Studien wurden bei Patienten mit schmerzhafter HIV-Neuropathie und unselektierten schmerzhaften Neuropathien durchgeführt [32, 33]. Die im Folgenden genannten „numbers needed to treat" (NNT) beziehen sich auf eine Schmerzreduktion $\geq 50 \%$.

Trizyklische Antidepressiva. Trizyklische Antidepressiva (z. B. Amitriptylin) sind gegen neuropathische Schmerzen wirksam, die errechnete NNT beträgt 2,6 (95\%-Konfidenzintervall [95 \%KI]: 2,2-3,3). Anticholinerge Nebenwirkungen (kognitive Verschlechterung, Delirien, Stürze, Harnverhalt, Obstipation) qualifizieren diese Substanzen im Alter als potenziell inadäquate Medikamente (PIM, [34]).

Selektive Serotonin- und Noradrenalin-Wiederaufnahmehemmer. Sehr unterschiedliche Ergebnisse zur Wirksamkeit zeigten selektive Serotonin-Wiederaufnahmehemmer (SSRI); sie werden daher nicht zur Behandlung neuropathischer Schmerzen empfohlen [32, 33]. Paroxetin ist mit einer NNT von 2,6 eine Ausnahme. Die Hemmung von Zytochrom-P450(CYP)2D6 ist unter dem Aspekt einer Polypharmakotherapie zu beachten. Von den Antidepressiva mit Serotonin- und Noradrenalin-Wiederaufnahmehemmung (selektive Noradrenalin-Wiederaufnahmehemmer, SNRI) wurden Venlafaxin und Duloxetin untersucht; beide waren wirksam [32, 33, 35]:

- Venlafaxin: NNT 5,2;

- Duloxetin: NNT: 4,9-5,2.

Im Rahmen der SSRI- und SNRI-Gabe ist auf eine Verlängerung der QT-Zeit zu achten. Hyponatriämie und Serotoninsyndrom (besonders in Kombination mit Opiaten) sind weitere unerwünschte Arzneimittelwirkungen.

Antiepileptika. Die natriumkanalblockierenden Antiepileptika Carbamazepin (NNT 3,3; 95 \%KI 2,5-9,4) und Phenytoin (NNT 2,1; $95 \%$-KI 1,5-3,6) zeigten ebenfalls positive Ergebnisse in der Behandlung neuropathischer Schmerzen [32, 33, 36]. Carbamazepin ist oft Auslöser einer Hyponatriämie; dies fällt unter dem Aspekt der im Alter häufigen Komedikation, z. B. mit 
Thiaziddiuretika oder SSRI, besonders ins Gewicht. Auch aufgrund vieler Interaktionen sind diese stark enzyminduzierenden Antiepileptika nicht zu empfehlen. Zudem führen sie vielfach zu kognitiver Beeinträchtigung und erhöhen das Risiko für eine Osteoporose.

Zu Lamotrigin existieren wenige, teils widersprüchliche Studien bei DPNP und HIV-assoziierter schmerzhafter Neuropathie [32, 33, 37]. Die Substanz ist im Alter meist gut verträglich.

Gabapentin, eine Untereinheit der N-Typ-kalziumkanalblockierenden Substanzen, bewirkte eine signifikante Reduktion neuropathischer Schmerzen (NNT 3,7; 95\%-KI, 2,4-8,3, [32, 33, 36]). Somnolenz, periphere Ödeme und Asthenie treten bei alten Patienten häufiger auf. Das verwandte Pregabalin hat sich ebenfalls als wirksam erwiesen (NNT 3,3-5,4, [32, 33, 36]). Bei beiden Substanzen ist eine Dosisreduktion bei abnehmender Nierenfunktion erforderlich.

Opiate. Aus der Gruppe der Opiate wurden Morphin, Oxycodon und Tramadol in kontrollierten Studien untersucht. Morphin reduzierte Schmerzen bei postherpetischer Neuralgie und DPNP (NNT 2,5). Retardiertes Oxycodon führte in einer mittleren Dosierung von $37 \mathrm{mg}$ bei Patienten mit DPNP zur signifikanten Schmerzreduktion. Tramadol, das neben einer schwachen Wirkung an Opiatrezeptoren auch die Serotonin- und Noradrenalinwiederaufnahme hemmt, zeigte ebenfalls eine signifikante Wirkung (NNT 3,4; $95 \%-\mathrm{KI}, 2,3-6,4$, [32, 33]).

Beim alten Patienten ist neben möglichen Interaktionen dem Risiko von Delirien besondere Aufmerksamkeit zu widmen. Morphin kann bei eingeschränkter Nierenfunktion akkumulieren. Eine antiemetische Therapie in der Initialphase erhöht die Akzeptanz ebenso wie eine adäquate Stuhlregulierung.

a-Liponsäure. Die i. v.-Gabe von a-Liponsäure (ALA) wurde an mehr als 1200 Patienten mit schmerzhafter DPNP untersucht. Eine rezente Metaanalyse kommt zum Schluss, dass die i. v.ALA-Gabe neuropathische Schmerzen bei DPNP in einem klinisch relevanten Ausmaß positiv beeinflusst (NNT 6,3; [38]).

Capsaicin. Das topische 8 \%ige Capsaicinpflaster war in Studien zur Behandlung neuropathischer Schmerzen wirksam; eine Publikation zeigte eine dem Pregabalin vergleichbare Wirksamkeit [39]. Aufgrund der geringen Nebenwirkungen ist dies eine interessante Therapieoption. Das Vorliegen eines fragilen Hautzustands beim Patienten limitiert die Anwendbarkeit.

Weitere Substanzen. Weitere Substanzen, für die keine ausreichenden Daten aus kontrollierten Studien vorliegen, sind u. a. Topiramat, Codidol (Dihydrocodein), transdermal verabreichtes Fentanyl, Mirtazapin, Piroxicam und Ibuprofen. Topiramat wird aufgrund von Nebenwirkungen (Gewichtsverlust, Anosmie, Ataxie, kognitive Störungen, Halluzinationen) nicht empfohlen. Piroxicam und Ibuprofen sind wegen der für nichtsteroidale Antirheumatika (NSAR) typischen Nebenwirkungen auf Magen und Nieren mit Zurückhaltung anzuwenden. Bei der Anwendung von transdermal appliziertem Fentanyl ist auf den intakten Zustand der Haut zu achten, um die adäquate Resorption zu gewährleisten.

\section{Nichtmedikamentöse Behandlungen}

Neben der Pharmakotherapie lassen sich neuropathische Schmerzen auch durch nichtmedikamentöse Behandlungen, wie Wärme- und Kälteanwendungen sowie Elektrotherapie positiv beeinflussen. Maßnahmen zur Kräftigung der unteren Extremitäten und zur Reduktion einer evtl. vorhandenen Sturzangst, sensomotorisches Training zur Verbesserung der Gleichgewichtsfähigkeit, Gangschulung und das Anpassen von Gehhilfen ergänzen das Spektrum. Weitere konservative Maßnahmen, wie z. B. der Einsatz von Peronäusschienen und anderer Orthesen, spielen in der symptomatischen Therapie von Neuropathien mit motorischen Beeinträchtigungen eine Rolle und können zu einem Gewinn an Lebensqualität beitragen.

Bei Neuropathien mit Prädisposition zu trophischen Störungen, z. B. der diabetischen Neuropathie, sind Maßnahmen zur Prävention von Ulzera erforderlich. Eine PNP ist in 85-90\% der Fälle an der Ätiologie des diabetischen Fußsyndroms beteiligt, die Untersuchung der Füße bei Menschen mit Diabetes ist daher obligat [19]. Exponierte Weichteilstellen mit erhöhter Druckbelastung wie über den Metatarsalköpfchen, Fersen, Fußaußenkanten oder Zehen, sind
Gabapentin bewirkte eine signifikante Reduktion neuropathischer Schmerzen

Retardiertes Oxycodon führte bei Patienten mit DPNP zur signifikanten Schmerzreduktion

Das 8 \%ige Capsaicinpflaster zeigte eine dem Pregabalin vergleichbare Wirksamkeit

Nichtsteroidale Antirheumatika sind wegen ihrer Nebenwirkungen mit Zurückhaltung anzuwenden

Neuropathien mit Prädisposition zu trophischen Störungen erfordern Maßnahmen der Ulzeraprävention 
Prädilektionsstellen für neuropathische Ulzerationen. Die Erhebung des peripheren Pulsstatus ist unverzichtbar, um eine Gefäßbeteiligung auszuschließen.

Bei alten Menschen spielt die Abklärung der funktionellen Fähigkeiten eine zentrale Rolle. Hierfür sind die Instrumente des Geriatrischen Assessments etabliert. Besonderes Augenmerk ist auf das Einschätzen von Mobilität und Sturzrisiko zu legen. In diesem Kontext ist auch die Abklärung einer möglichen Osteoporose essenziell, um ggf. prophylaktische und therapeutische Maßnahmen ergreifen zu können.

\section{Fazit für die Praxis}

- Polyneuropathien sind häufige Erkrankungen des höheren Lebensalters.

- Sie können die Mobilität des Betroffenen beeinträchtigen und das Sturzrisiko erhöhen.

- Subjektiv steht der neuropathische Schmerz häufig im Vordergrund.

- Das Spektrum möglicher Ursachen ist breit; Diabetes mellitus, Niereninsuffizienz, Malignome und monoklonale Gammopathien unklarer Signifikanz finden sich häufig.

- Polyneuropathien können Erstmanifestation systemischer Erkrankungen sein.

- Anamnese und klinische Untersuchung werden je nach vermuteter Ätiologie um elektrophysiologische- und Laboruntersuchungen ergänzt.

- Die Therapie des neuropathischen Schmerzes erfordert den Einsatz von Antiepileptika, Antidepressiva und Analgetika.

- Der Abklärung der funktionellen Fähigkeiten kommt entscheidende Bedeutung in der Planung von nichtmedikamentösen Therapiemaßnahmen und der Hilfsmittelversorgung zu.

\section{Korrespondenzadresse}

Prim. Univ.-Prof. Dr. B. Iglseder

Universitätsklinik für Geriatrie, Christian-Doppler-Klinik, Universitätsklinikum Salzburg, Paracelsus Medizinische Privatuniversität

Ignaz-Harrer-Str. 79, 5020 Salzburg, Österreich

b.iglseder@salk.at

Open access funding provided by Paracelsus Medical University.

\section{Einhaltung ethischer Richtlinien}

Interessenkonflikt. W. Löscher und B. Iglseder geben an, dass kein Interessenkonflikt besteht.

Dieser Beitrag beinhaltet keine von den Autoren durchgeführten Studien an Menschen oder Tieren.

Open Access. Dieser Artikel wird unter der Creative Commons Namensnennung 4.0 International Lizenz (http://creativecommons. org/licenses/by/4.0/deed.de) veröffentlicht, welche die Nutzung, Vervielfältigung, Bearbeitung, Verbreitung und Wiedergabe in jeglichem Medium und Format erlaubt, sofern Sie den/die ursprünglichen Autor(en) und die Quelle ordnungsgemäßnennen, einen Link zur Creative Commons Lizenz beifügen und angeben, ob Änderungen vorgenommen wurden.

\section{Literatur}

1. Arnold N, Harriman DG (1970) The incidence of abnormality in control human peripheral nerves studied by single axon dissection. J Neuro Neurosurg Psychiatry 33(1):55-61

2. Low PA, Dyck PJ (1978) Splanchnic preganglionic neurons in man. III. Morphometry of myelinated fibers of rami communicantes. JNeuropathol Exp Neurol 37(6):734-740

3. Vrancken $A F$, Franssen $H$, Wokke $\mathrm{JH}$, Teunissen LL, Notermans NC (2002) Chronic idiopathic axonal polyneuropathy and successful aging of the peripheral nervous system in elderly people. Arch Neurol 59(4):533-540

4. Goble DJ, Coxon JP, Wenderoth N, Van IA, Swinnen SP (2009) Proprioceptive sensibility in the elderly: degeneration, functional consequences and plastic-adaptive processes. Neurosci Biobehav Rev 33(3):271-278

5. Suetterlin KJ, Sayer AA (2014) Proprioception: where are we now? A commentary on clinical assessment, changes across the life course, functional implications and future interventions. Age Ageing 43(3):313-318

6. Callaghan B, Kerber K, Langa KM, Banerjee M, Rodgers A, McCammon Ret al (2015) Longitudinal patientoriented outcomes in neuropathy: importance of early detection and falls. Neurology 85(1):71-79

7. Clark BC, Manini TM (2012) What is dynapenia? Nutrition 28(5):495-503

8. Hanewinckel R, van Oijen $M$, Ikram MA, van Doorn PA (2016) The 
epidemiology and risk factors of chronic polyneuropathy. Eur J Epidemiol 31(1):5-20

9. Visser NA, Notermans NC, Linssen $\mathrm{RS}$, van den Berg LH, Vrancken AF (2015) Incidence of polyneuropathy in Utrecht, the Netherlands. Neurology 84(3):259-264

10. Dyck PJ, Dyck PJ, Grant IA, Fealey RD (1996) Ten steps in characterizing and diagnosing patients with peripheral neuropathy. Neurology 47(1):10-17

11. Staff NP, Windebank AJ (2014) Peripheral neuropathy due to vitamin deficiency, toxins, and medications. Continuum Lifelong Learn Neurol 20(5 Peripheral Nervous System Disorders):1293-1306

12. CorraoG,Zambon A, Bertu L, Botter E, Leoni O, Contiero P (2004) Lipid lowering drugs prescription and the risk of peripheral neuropathy: an exploratory case-control study using automated databases. $\mathrm{J}$ Epidemiol Community Health 58(12):1047-1051

13. Richardson PG, Sonneveld P, Schuster MW, Stadtmauer EA, Facon T, Harousseau JL et al (2009) Reversibility of symptomatic peripheral neuropathy with bortezomib in the phase III APEX trial in relapsed multiple myeloma: impact of a dose-modification guideline. $\mathrm{Br} \mathrm{J}$ Haematol 144(6):895-903

14. Thai XC, Bruno-Murtha LA (2006) Bell's palsyassociated with linezolid therapy: case report and review of neuropathic adverse events. Pharmacotherapy 26(8):1183-1189

15. Taylor PK (1984) Non-linear effects of age on nerve conduction in adults. J Neurol Sci 66(2-3):223-234

16. Mallik A, Weir Al (2005) Nerve conduction studies: essentials and pitfalls in practice. J Neurol Neurosurg Psychiatry 76(Suppl 2):ii23-ii31

17. England JD, Gronseth GS, Franklin G, Carter GT, Kinsella LJ, Cohen JA et al (2009) Evaluation of distal symmetric polyneuropathy: the role of laboratory and genetic testing (an evidence-based review). Muscle Nerve 39(1):116-125

18. England JD, Gronseth GS, Franklin G, Carter GT, Kinsella LJ, Cohen JA et al (2009) Evaluation of distal symmetric polyneuropathy: the role of autonomic testing, nerve biopsy, and skin biopsy (an evidence-based review). Muscle Nerve 39(1):106-115

19. Callaghan BC, Cheng HT, Stables CL, Smith AL, Feldman EL (2012) Diabetic neuropathy: clinical manifestations and current treatments. Lancet Neurol 11(6):521-534

20. Dyck PJ, Norell JE, Dyck PJ (1999) Microvasculitis and ischemia in diabetic lumbosacral radiculoplexus neuropathy. Neurology 53(9):2113-2121

21. Ropper AH, Gorson KC (1998) Neuropathies associated with paraproteinemia. N Engl J Med 338(22):1601-1607

22. Dimachkie MM, Barohn RJ (2013) Guillain-Barre syndrome and variants. Neurol Clin 31(2):491-510

23. Hattori N, Misu K, Koike H, Ichimura M, Nagamatsu M, Hirayama M et al (2001) Age of onset influences clinical features of chronic inflammatory demyelinating polyneuropathy. J Neurol Sci 184(1):57-63

24. Collins MP, Dyck PJ, Gronseth GS, Guillevin L, Hadden RD, Heuss D et al (2010) Peripheral Nerve Society Guideline on the classification, diagnosis, investigation, and immunosuppressive therapy of nonsystemic vasculitic neuropathy: executive summary. JPeripher Nerv Syst 15(3):176-184

25. Michet CJ (1990) Epidemiology of vasculitis. Rheum Dis Clin North Am 16(2):261-268

26. Monforte R, Estruch R, Valls-Sole J, Nicolas J, Villalta J, UrbanoMarquez A (1995) Autonomic and peripheral neuropathies in patients with chronic alcoholism. A doserelated toxic effect of alcohol. Arch Neurol 52(1):45-51

27. Antoine JC, Mosnier JF, Absi L, Convers $\mathrm{P}$, Honnorat J, Michel D (1999) Carcinoma associated paraneoplastic peripheral neuropathies in patients with and without antionconeural antibodies. J Neurol Neurosurg Psychiatry 67(1):7-14

28. Vedeler CA, Antoine JC, Giometto B, Graus F, Grisold W, Hart IK et al (2006) Management of paraneoplastic neurological syndromes: report of an EFNS task force. Eur J Neurol 13(7):682-690

29. Ceravolo R, Cossu G, di Bandettini PM, Santoro L, Barone P, Zibetti
M et al (2013) Neuropathy and levodopa in Parkinson's disease: evidence from a multicenter study. Mov Disord 28(10):1391-1397

30. Chung SA, Seo P (2009) Advances in the use of biologic agents for the treatment of systemic vasculitis. Curr Opin Rheumatol 21(1):3-9

31. Dinser $R$, Jendro $M C$, Schnarr S, Zeidler H (2005) Antibiotic treatment of Lyme borreliosis: what is the evidence? Ann Rheum Dis 64(4):519-523

32. Baron R, Binder A, Wasner G (2010) Neuropathic pain:diagnosis, pathophysiological mechanisms, and treatment. Lancet Neurol 9(8):807-819

33. Finnerup NB, Attal N, Haroutounian S, McNicolE, Baron R, Dworkin RHet al (2015) Pharmacotherapy for neuropathic pain in adults: a systematic review and meta-analysis. Lancet Neurol 14(2):162-173

34. Holt S, Schmiedl S, Thurmann PA (2010) Potentially inappropriate medications in the elderly: the PRISCUS list. Dtsch Arztebl Int 107(31-32):543-551

35. Lunn MP, Hughes RA, Wiffen PJ (2014) Duloxetine for treating painful neuropathy, chronic pain or fibromyalgia. Cochrane Database Syst Rev:. doi:10.1002/14651858. cd007115.pub3

36. Wiffen PJ, Derry $S$, Moore RA, Aldington D, Cole P, Rice AS et al (2013) Antiepileptic drugs for neuropathic pain and fibromyalgia - an overview of Cochrane reviews. Cochrane Database Syst Rev:. doi:10.1002/14651858.cd010567. pub2

37. Wiffen PJ, Derry S, Moore RA (2013) Lamotrigine for chronic neuropathic pain and fibromyalgia in adults. Cochrane Database Syst Rev:. doi:10.1002/14651858. cd006044.pub4

38. Mcllduff CE, Rutkove SB (2011) Critical appraisal of the use of alpha lipoic acid (thioctic acid) in the treatment of symptomatic diabetic polyneuropathy. Ther Clin Risk Manag 7:377-385

39. Haanpaa M, Cruccu G, Nurmikko TJ, McBride WT, Docu AA, Bosilkov A et al (2016) Capsaicin $8 \%$ patch versus oral pregabalin in patients with peripheral neuropathic pain. Eur JPain 20(2):316-328 
Teilnahme am zertifizierten Kurs auf CME.SpringerMedizin.de

- Der Teilnahmezeitraum beträgt 12 Monate, den Teilnahmeschluss finden Sie online beim CME-Kurs.

- Fragen und Antworten werden in zufälliger Reihenfolge zusammengestellt.

- Pro Frage ist jeweils nur eine Antwort zutreffend.

- Für eine erfolgreiche Teilnahme müssen 70 \% der Fragen richtig beantwortet werden.

? Welches der folgenden Arzneimittel soll zur Behandlung neuropathischer Schmerzen beim alten Menschen vorrangig eingesetzt werden?

O Diclofenac

O Gabapentin

○ Citalopram

O Vitamin $B_{6}$

○ Amitriptylin

Polyneuropathien im Rahmen eines Diabetes mellitus (DPNP) sind im Alter häufig. Welche der folgenden Aussagen zu DPNP trifft am besten zu?

O Die diabetische Amyotrophie betrifft überwiegend Menschen mit Typ-1-Diabetes.

O Schmerzen im Orbitalbereich schließen eine diabetische Ophthalmoplegie weitgehend aus.

○ Die Prävalenz einer DPNP ist bei jüngeren Patienten höher.

○ Am häufigsten ist die distal symmetrische, sensibel betonte Neuropathie.

○ Lähmungen und Atrophien der Beckengürtel- und Oberschenkelmuskulatur finden sich bei der diabetischen Amyotrophie nur ausnahmsweise.

? Welche Untersuchung ist zur Abklärung chronischer distal symmetrischer vorwiegend sensibler Neuropathien nicht indiziert?

O Bestimmung von Vitamin $B_{12}$

O Bestimmung von $\mathrm{HbA}_{1 \mathrm{C}}$

O Serumelektrophorese

○ Bestimmung des Serum-Nüchtern-Glucose-Spiegels

O Magnetresonanztomographie der Wirbelsäule
Bei einer 74-jährigen Patientin wird eine Mononeuritis multiplex diagnostiziert. Welche der folgenden Ursachen ist am wahrscheinlichsten?

O Chronisch inflammatorische demyelinisierende Neuropathie

○ Vaskulitis

O Alkoholerkrankung

O Chemotherapie - induzierte Neuropathie

Kryptogene axonale Polyneuropathie

Welche der folgenden Aussagen trifft für Paraproteinämien als Ursache von PNP am besten zu?

O Im Alter finden sich vermehrt Paraproteinämien (monoklonale Gammopathien).

O Im Verlauf entwickeln fast alle dieser Patienten eine systemische Erkrankung wie multiples Myelom oder M. Waldenström.

○ Bezüglich der Immunglobulinklassen überwiegt mit mehr als $50 \%$ der lg-GTyp.

O Eine Serumelektrophorese mit Immunfixation ist für die Diagnosestellung in der Regel nicht erforderlich.

O Initial stehen autonome Symptome im Vordergrund.

Welches der folgenden Symptome passt am wenigsten zur Diagnose einer PNP?

○ Parästhesie

O Hypästhesie

O Sockenförmige Schmerzen

O Gesteigerte Muskeleigenreflexe

$\bigcirc$ Unsicherheit beim Gehen in der Dunkelheit
? Das PNS zeigt im Laufe des Alternsprozesses Veränderungen. Welche der folgenden Aussagen trifft am wenigsten zu?

Das Berührungsempfinden zeigt mit dem Alter zunehmend eine höhere Schwelle.

○ Die Propriozeption leidet durch eine Reduktion der Mechanorezeptoren der distalen Gelenke und der Haut.

○ Eine Abschwächung der Muskeleigenreflexe imponiert besonders an den unteren Extremitäten distal betont.

O Die neurophysiologisch messbaren sensiblen Nervenleitgeschwindigkeiten zeigen im Alter keine wesentlichen Änderungen.

O An den Beinen findet sich häufig eine distal betonte Reduktion des Vibrationsempfindens.

Zu den positiven sensiblen Symptomen einer PNP zählt man:

○ Muskelkrämpfe

O Faszikulationen

O Myokymien

O Dysästhesien

O Hypästhesien

Welches der folgenden Medikamente führt nach derzeitigem Kenntnisstand nicht zu einer PNP?

O Amiodaron

Cisplatin

O Simvastatin

O Amlodipin

O Nitrofurantoin 
Welche der folgenden Aussagen zur alkoholisch bedingten PNP trifft am ehesten zu?

Elektrophysiologisch finden sich Zeichen der Demyelinisierung.

O Sie ist vorwiegend Folge einer direkten neurotoxischen Wirkung.

O Sie beginnt meist proximal symmetrisch.

O Die Symptomatik beginnt mit verminderter Schmerzwahrnehmung in den betroffenen Gebieten.

○ Am Anfang steht die Muskelschwäche im Vordergrund; sensible Störungen treten erst spät im Verlauf auf.

\section{e.Curriculum Innere Medizin}

Vorhofflimmern - Interaktive Fälle zur Leitlinie - Update

So nehmen Sie teil:

1. Anmelden: Bitte melden Sie sich mit Ihrem SpringerMedizin-Account an. Sollten Sie noch nicht angemeldet sein, können Sie sich hier registrieren:

CME.SpringerMedizin.de

2. Kurs auswählen und bearbeiten: Mit "Starten" können Sie die Fortbildung bearbeiten und die MC-Fragen des Fragebogens beantworten.

3. CME-Punkte sammeln: Mit 70\% richtig beantworteten Fragen haben Sie bestanden. Bei hinterlegter EFN werden die Punkte an den EIV übermittelt.

Springer Medizin

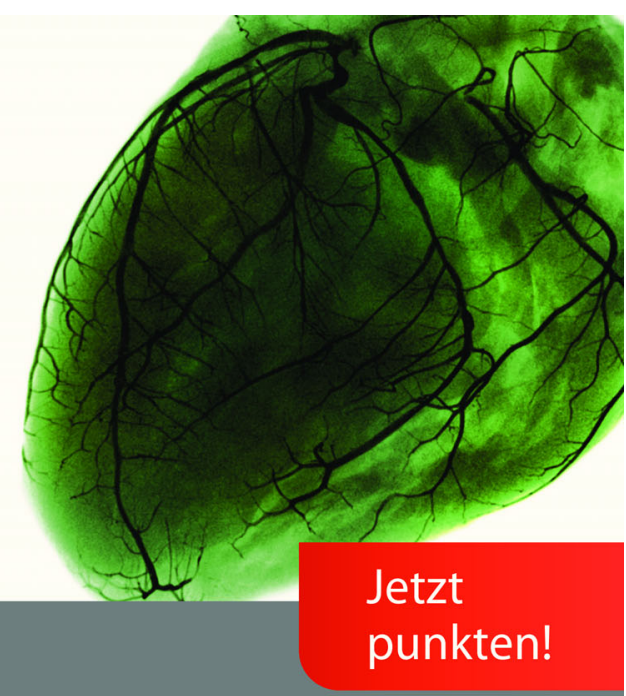

Das Fortbildungsmodul ist:

- mit 3 CME-Punkten zertifiziert,

- interaktiv und multimedial,

- fallbasiert und leitlinienorientiert,

- exklusiv für DGIM-Mitglieder und e.Med-Abonnenten.

www.SpringerMedizin.de/ecurriculum-innere-medizin 